\title{
Escala de Competencias Emocionales de Abuelos y Abuelas (ECEA)
}

\author{
Scale on Grandparents' Emotional Competences (GPEC)
}

\author{
Verónica García Díaz y Raquel-Amaya Martínez-González ${ }^{2}$
}

\begin{abstract}
Resumen
La ausencia de estudios y de herramientas que analicen la percepción que abuelos y abuelas tienen de su competencia emocional para con sus nietos y nietas, hace que surja la necesidad de diseñar y, posteriormente, validar un instrumento que aporte información al respecto. Para ello se ha contando con una muestra de 140 abuelos y 210 abuelas que cuidan de sus nietos y nietas. En la validación de la escala se trabaja con dos submuestras, ejecutando con una de ellas un análisis factorial exploratorio y con otra un análisis factorial confirmatorio. Como resultado se obtiene una escala de 10 ítems distribuidos en cuatro factores que explican el $45.25 \%$ de la varianza, con índices de fiabilidad adecuados: estrés, seguridad personal, dificultades para conciliar el cuidado de los nietos con la vida personal y autorregulación emocional. La escala presenta valores de validez concurrente aceptables con la satisfacción y eficacia percibida en el desarrollo de las funciones educativas. El estudio explora, asimismo, resultados diferenciales de las competencias emocionales de los abuelos y abuelas en función del sexo, edad y línea de parentesco.
\end{abstract}

Palabras clave: escala, competencias emocionales, abuelos, análisis factorial, sexo, edad, línea de parentesco

\begin{abstract}
The lack of studies and tools that analyze the perception that grandparents have of their emotional competence towards their grandchildren, makes it necessary to design and then validate an instrument that provides information about it. To this end, a sample composed of 140 male grandparents and 210 grandmothers who take care of their grandchildren took part in the study. In the validation of the scale two subsamples were considered, performing with one of them an exploratory factor analysis and with the other one a confirmatory factor analysis. As a result, a 10 item scale was obtained, distributed in four factors that explain $45.25 \%$ of the variance, with adequate reliability indexes: stress, assertiveness, difficulties to conciliate the care of grandchildren with personal time and emotional self-regulation. The scale presents acceptable concurrent validity values with perceived satisfaction and efficacy in the development of educational functions. The study also explores differential results of emotional competencies of grandparents based on gender, age and kinship.
\end{abstract}

Keywords: scale, grandparents emotional competences, factor analysis, sex, age, kinship

\footnotetext{
${ }^{1}$ Doctoranda, Universidad de Oviedo, España. Correo: veronica.pdg@gmail.com

${ }^{2}$ Catedrática de Universidad Acreditada y Profesora Titular, Universidad de Oviedo, España.

Revista Iberoamericana de Diagnóstico y Evaluación - e Avaliação Psicológica. RIDEP · No49 · Vol.4 · 107-120 · 2018

ISSN: 1135-3848 print /2183-6051online
} 


\section{Introducción}

Varios autores han puesto de manifiesto la escasa atención que ha prestado la literatura científica al papel que en la actualidad asumen abuelos y abuelas en la familia (Osuna, 2006; Pinazo, 1999; Triadó et al., 2008c). Ello hace que surja la necesidad de estudiar la valiosa función que desempeñan en el cuidado y educación de sus nietos y nietas y la variedad de situaciones y competencias personales que la condicionan (García, Pérez, \& Martínez, 2014; Megías \& Ballesteros, 2011; Roa \& Vacas, 2000). Ello está en relación con la evolución que la familia ha venido experimentando a lo largo del tiempo. Los autores coinciden en señalar que la familia es la primera institución socializadora (Martínez, 1996; Menéndez, 2001a, 2001b; Muñoz, 2005; Perea, 2006; Isaza, 2013), a quien la sociedad encomienda la función insustituible de servir como ámbito básico de aprendizaje, que favorezca el desarrollo óptimo e integral de la infancia. Se la considera unidad básica de la sociedad y contexto que contribuye al proceso de individualización y socialización de sus miembros más pequeños. Desde un modelo psicopedagógico, Martínez (1996) entiende la familia como un contexto en el que, de darse las condiciones adecuadas, padres y madres pueden facilitar el desarrollo de capacidades, habilidades, valores, motivaciones, y la estabilidad emocional y afectiva en sus hijos e hijas.

No obstante, y puesto que necesita adaptarse a la pluralidad de cambios que, a distintos niveles, se han dado en la sociedad contemporánea (Gimeno, 1999; Iglesias de Ussel, 1998; Palacios \& Rodrigo, 1998; Rodríguez \& Menéndez, 2003; Valdivia, 2008), el sistema familiar puede llegar a ser vulnerable y encontrar obstáculos para desarrollar adecuadamente esta función social mencionada. Algunos de esos cambios, como la incorporación de la mujer al trabajo, la aparición de nuevas formas familiares, la escasez de políticas sociales dirigidas a la conciliación entre la vida laboral, familiar y personal, entre otros, hacen que no siempre resulte fácil la atención a los hijos e hijas, y que padres y madres se vean en la necesidad de delegar parte de su responsabilidad educativa en otros agentes socializadores, como los abuelos y abuelas
(Gomila, 2005; González, 2007; Meil \& RogeroGarcía, 2014; Mestre, 2011; Pérez, 2007; Tobío, 2002, 2012, 2013).

Badenes y López (2011) señalan que cuando padres y madres no pueden desempeñar correctamente las funciones de crianza y educación esperadas, es necesaria la implicación de estos miembros de la familia extensa. De ahí que Triadó (2015) apunte que el ejercicio de estas funciones sustitutorias por parte de abuelos y abuelas, ha hecho resurgir su importancia dentro de la familia. Su implicación actual incluye el cumplimiento de tareas que van más allá de la atención básica y de la tradicional transmisión de valores y experiencias a sus nietos y nietas, asumiendo ahora mayores responsabilidades; González, De la Fuente y González (2015) sostienen que éstas se traducen en un protagonismo compartido con los padres y madres en el desarrollo afectivo y personal de sus pequeños. Al respecto, y por sus posibles efectos en la edad adulta, Frías, Díaz y Reyes (2011) y Maglio, Molina, Raimundi, González y Schmidt (2014) hacen hincapié en la importancia del apoyo social percibido, pues todo ello contribuye al desarrollo óptimo e integral al que se aludía con anterioridad.

Con referencia a las funciones que desarrollan en la actualidad los abuelos y abuelas, la mayoría de autores que centran sus investigaciones en este tema, como González, González, De la Fuente, Marquínez y González (2010), Pinazo (1999), Triadó, Martínez y Villar (2000), Triadó, Villar, Solé, Osuna \& Pinazo (2005) y Rico, Serra y Viguer (2001), hacen mención a una pluralidad de roles y estilos, ya sea estudiándolos desde la perspectiva de los propios abuelos y abuelas o desde la de sus nietos y nietas. González et al. (2010) y Rico, Serra y Viguer (2001), hablan de abuelo y abuela cuidador, historiador, transmisor de conocimientos y de valores, apoyo emocional, etc. Por su parte, Triadó, Martínez y Villar (2000) y Triadó et al. (2005), recogiendo información en sus investigaciones con la adaptación de la escala Grandparent Meaning Scale (GMS) de Van Rast, Verschueren \& Marcoen (1995), destacan las funciones de cuidador, alianza fiable, fuente de afecto y ayuda emocional, etc. Pinazo (1999) hace una revisión teórica sobre este tema citando autores que proponen tipologías relevantes de 
abuelos y abuelas (independiente, pasivo, activo, formal, distante, etc.), entre ellos: Cherlin y Furstenberg $(1985,1986)$, Neugarten y Weinstein (1964) o Wood y Robertson (1976).

En otros trabajos, Triadó et al. (2008a, 2008b, 2008c), utilizando técnicas de recogida de información de elaboración propia, enumeran y describen las tareas que abuelos y abuelas realizan con sus nietos y nietas (instrumentales, de ocio, disciplina, etc.), contemplando también la satisfacción y la dificultad con que se vive este rol. En torno a esta última idea, Hoyuelos (2004) y Osuna (2006) reconocen la satisfacción y el optimismo como principales sentimientos asociados a esta función. Osuna (2006) señala que esta satisfacción está asociada a un sentimiento de futuro y optimismo, y Hoyuelos (2004), añade que abuelos y abuelas sienten emoción y felicidad cuando cuidan de sus nietos y nietas; si bien, según éste, pueden verse en la tesitura de encajar en moldes y expectativas de un rol que está poco definido y es confuso. Por su parte, Bernad (2012), Osuna (2006), Rico, Serra y Viguer (2001), entre otros, sostienen que hay factores que, por su influencia en el desempeño de este rol $\mathrm{y}$, por tanto, también en la visión que abuelos y abuelas tienen al respecto, requieren estudio $\mathrm{y}$ atención.

Así pues, en la actualidad se asume que abuelos y abuelas tienen una importancia clave en la provisión de apoyo y estabilidad emocional a sus nietos y nietas cuando, por distintos motivos, sus padres y madres no pueden dedicarles el tiempo y la atención que se espera del ejercicio del rol parental. De hecho, Triadó (2105) señala que en muchas familias son los abuelos y abuelas quienes asumen la responsabilidad afectiva, educativa e incluso económica de sus nietos y nietas, proporcionándoles afecto, tiempo, seguridad y protección durante su infancia y adolescencia. Para ello se requiere contar con las competencias adecuadas. De acuerdo con Bisquerra y Pérez (2007), las competencias, en general, implican la posibilidad de poner adecuadamente en ejercicio capacidades, habilidades, conocimientos y actitudes que sean idóneas para lo que se precisa en cada circunstancia.

En la revisión de estudios realizada se ha observado que las investigaciones se centran en funciones, roles, estilos, tipologías, tareas, consecuencias, etc., pero no se ha encontrado ninguna que profundice en las competencias emocionales que perciben que tienen los propios abuelos y abuelas para ejercer este cometido, ni técnicas de recogida de información validadas y específicas al respecto. De ahí que este estudio se plantee dos objetivos: 1) diseñar una escala específica sobre competencias emocionales de abuelos y abuelas con respecto al cuidado de sus nietos y nietas, analizando su estructura y sus características técnicas; y 2) identificar dichas competencias en una muestra de abuelos y abuelas, considerando, además, posibles diferencias en función del sexo, de la edad y de la línea de parentesco.

\section{Método}

\section{Participantes}

En el estudio han participado 350 personas, 210 abuelas (60\%) y 140 abuelos (40\%), la mayoría españolas $(99.2 \%)$ y residentes en municipios del centro de Asturias (España) (99.1\%). El 98.6\% vive en su propia casa y sólo el $1.4 \%$ en casa de otros familiares $(1.1 \%)$ o de su hijo o hija (0.3\%). En cuanto a su edad, el $66.1 \%$ tiene entre 40 y 64 años y el $33.9 \% 65$ o más. Del estado civil, se destaca que el $86 \%$ está casado, el $7.1 \%$ viudo y el $6.3 \%$ separado o divorciado, si bien un $0.6 \%$ se identifica con otros estados. Un predominante porcentaje vive acompañado (90.2\%) y otro $9.8 \%$ solo. Respecto al nivel de estudios, el $61.1 \%$ cuenta con estudios primarios, el $23.4 \%$ con estudios secundarios, bachillerato y/o formación profesional y el $9.9 \%$ con estudios universitarios; el 5.6\% manifiesta que no tiene estudios. De su situación laboral, se recoge que el $84.2 \%$ no desempeña un trabajo remunerado fuera de casa, frente al $15.8 \%$ que señala lo contrario. En referencia a ingresos familiares, el $56.2 \%$ percibe más de 1800 euros al mes, el $21.2 \%$ entre 600 y 1200 , el $19.7 \%$ entre 1201 y 1800 y el $2.9 \%$ menos de 600 euros. En la Tabla 1 se resumen las características socio-demográficas de la muestra.

Un $54.9 \%$ cuida de dos nietos y nietas, un $36 \%$ de uno, un $7.1 \%$ de tres y un $2 \%$ de cuatro. Del total de nietos y nietas por quienes los abuelos y abuelas responden el cuestionario, $213(60.9 \%)$ son niños y 137 (39.1\%) niñas, cuya edad está 
comprendida mayoritariamente $(84.9 \%)$ entre uno y diez años; un $12.3 \%$ tiene entre once y quince años y el $2.9 \%$ menos de un año. El $60.9 \%$ de la muestra obtenida son abuelos y abuelas maternos, por parte de hija, y el $39.1 \%$ paternos, por parte de hijo.

Tabla 1. Características socio-demográficas de la muestra

\begin{tabular}{lc}
\hline Variables & Porcentajes \\
\hline Sexo & \\
Abuelas & $60 \%$ \\
Abuelos & $40 \%$ \\
Hogar de residencia & \\
$\quad$ Vive en su propia casa & $98.6 \%$ \\
$\quad$ Vive acompañado/a & $90.2 \%$ \\
Edad & \\
Entre 40 y 64 años & $66.1 \%$ \\
$\quad 65$ años o más & $33.9 \%$ \\
Estado civil & \\
$\quad$ Casado & $86 \%$ \\
Viudo & $7.1 \%$ \\
Separado o divorciado & $6.3 \%$ \\
Otros estados & $0.6 \%$ \\
Nivel de estudios & \\
Estudios primarios & $61.1 \%$ \\
Estudios secundarios, & $23.4 \%$ \\
bachillerato y/o formación & \\
profesional & \\
Estudios universitarios & \\
Sin estudios & $9.9 \%$ \\
Situación laboral & $5.6 \%$ \\
No desempeña trabajo & \\
remunerado fuera de casa & \\
Sí desempeña trabajo & \\
remunerado fuera de casa & \\
\hline
\end{tabular}

\section{Instrumento}

Tras efectuar una revisión de la literatura científica sobre este tema en bases documentales como ERIC, ISOC, SCOPUS, WOS, no se encontró un instrumento adecuado que permitiera obtener la información necesaria para responder al objetivo de este trabajo; por ello, se decidió construir un cuestionario propio a tal efecto. Para su elaboración se siguieron las fases indicadas en la literatura especializada sobre este tipo de técnicas (De Lara-Guijarro \& BallesterosVelázquez, 2007; Gil, 2011; Muñiz, 2000). En la primera, se construyó un borrador inicial una vez establecidas varias dimensiones de estudio; estas se identificaron tras efectuar un análisis de contenido sobre una selección de artículos centrados en el tema de investigación, que han sido referenciados en la introducción de este artículo. Concretamente, se tomó en consideración las ideas más relevantes que señalaban los autores sobre el cuidado y educación de los nietos y nietas, así como nuestras propias ideas sobre los aspectos que necesitaban mayor investigación. Se establecieron así cuatro dimensiones de análisis que incluyen aspectos relativos a la competencia emocional con que los abuelos y abuelas afrontan su rol de cuidadores y educadores de sus nietos y nietas y a la percepción que tienen de sus habilidades educativas para desempeñar este rol: 1) estrés, 2) seguridad, 3) dificultad para conciliar esta función con sus actividades personales y 4) autorregulación emocional en el ejercicio de su rol educativo. Se identificaron escalas dirigidas a padres y madres ya construidas sobre competencias parentales que, adaptando algunos de sus ítems hacia los abuelos y abuelas, pudieron ser tomadas como punto de partida. Estas escalas son: 1) Escala de Percepción de Competencia Parental, PSOC, de Johnston y Mash (1989); 2) Escala de Evaluación Parental, EEP, de FarKas (2008); y 3) Escala de Competencias Parentales, Emocionales y Sociales, de Martínez (2009). Asimismo, se redactaron otros ítems de nueva creación sobre las dimensiones mencionadas para configurar el primer borrador del cuestionario.

Una segunda fase consistió en la revisión de este borrador por un grupo de investigadores especializados en métodos de investigación y en orientación familiar del grupo de investigación sobre Intervención Educativa en el Ámbito Familiar, Escolar y Social (IEFES), de la Universidad de Oviedo; con ello se pretendía valorar tanto la relevancia del contenido y dimensiones incluidas, como la calidad de los ítems. Tras este proceso, varios ítems fueron modificados, otros nuevos fueron incluidos $y$ algunos otros fueron eliminados. En la tercera fase se realizó una consulta a través de la técnica Delphi (Cabero e Infante, 2014; León y Montero, 2003) a un total de diez expertos tanto en educación familiar como en elaboración de cuestionarios, para validar el nuevo instrumento; se trataba de investigadores externos al grupo IEFES, de varias universidades, y, por tanto, distintos a los consultados con anterioridad. Se les pidió que valoraran la representatividad, relevancia, coherencia y claridad de los ítems en una escala desde 1-Nada hasta 4-Mucho. La media obtenida de la información suministrada 
por estos expertos en los aspectos evaluados oscila entre 3.60 y 3.70. La plantilla incluía también una pregunta abierta para recoger sus valoraciones cualitativas. El análisis de contenido efectuado sobre sus respuestas, permitió mejorar la redacción de algunos ítems y reordenarlos.

La cuarta fase consistió en aplicar el cuestionario de modo piloto a 10 personas (4 abuelos y 6 abuelas), de entre 40 y 64 años (77.8\%) y de más de 65 (22.2\%), que cuidan de sus nietos y nietas. Con ello se pretendía valorar si el cuestionario resultaba claro, coherente y fácil de responder. Las aportaciones sugeridas, fundamentalmente sobre la comprensión de algún ítem, se incorporaron en el cuestionario y se sometió éste a una nueva revisión por parte de tres miembros del grupo de investigación IEFES de la Universidad de Oviedo. La última versión constaba de 24 ítems con respuesta en escala Likert de cuatro alternativas (1-Nunca, 2-A Veces, 3-Casi Siempre y 4-Siempre), de los cuales 20 han sido adaptados de las escalas mencionadas ya publicadas sobre competencias parentales y sobre percepción de capacidad parental: 1) Escala de Percepción de Competencia Parental, PSOC, de Johnston y Mash (1989), de la que se adaptaron 8 ítems del tipo "Una de las cosas más difíciles de ser abuelo/abuela es saber si lo estoy haciendo bien o no"; 2) Escala de Evaluación Parental, EEP, de FarKas (2008), de la que se adaptaron 7 ítems del tipo "Creo que soy un buen abuelo/abuela y que cumplo adecuadamente con mis funciones"; y 3) Escala de Competencias Parentales, Emocionales y Sociales, de Martínez (2009), de la que se adaptaron 5 ítems del tipo "Sé relajarme y controlar mis emociones ante mi nieto/a". Asimismo, la escala final construida incluía otros 4 nuevos ítems de interés para el tema, del tipo "Tener que cuidar de mi nieto/a me impide hacer cosas o ir a sitios que me gustaría". Se incluyeron también variables sociodemográficas que aportan datos de interés tanto del abuelo y abuela, como del nieto o nieta bajo su cuidado. Además, en la versión definitiva del cuestionario se añadieron dos ítems en un bloque complementario, con la pretensión de que funcionaran como variables criterio externas de eficacia y satisfacción en el desempeño del rol socializador, respectivamente, y permitieran analizar la validez concurrente. Los dos ítems son:
"Me manejo muy bien con las tareas que tengo que hacer con mi nieto/a" (eficacia) y "Hacer de abuelo/a es tan satisfactorio como yo esperaba" (satisfacción); tal como ha puesto de manifiesto la literatura consultada, ambos criterios representan adecuadamente las competencias emocionales y educativas implicadas en el desempeño del rol socializador de los abuelos y abuelas.

\section{Procedimiento}

Se ha recogido información de 350 abuelos y abuelas seleccionados mediante un muestreo no probabilístico incidental de "bola de nieve" (snowball sampling) (Goodman, 1961), atendiendo a su voluntariedad para participar en el estudio. En concreto, se invitó a abuelos y abuelas que estuvieran ejerciendo funciones de cuidado y educación con al menos uno de sus nietos o nietas. Asimismo, y para garantizar cierta heterogeneidad, se buscó que participasen personas con edades comprendidas desde 40 años a 65 o más. Estos abuelos y abuelas distribuyeron copias a otros de su entorno, y éstos, a su vez, a otros, hasta obtener el número necesario. Se entregaron sobres que contenían una carta de presentación de la investigación, un cuestionario para el abuelo y otro cuestionario para la abuela. Una vez cumplimentados, éstos lo introducían en el sobre, se lo entregaban a la persona de referencia, y ésta a la investigadora. Para garantizar un tamaño muestral suficientemente grande que permitiera efectuar los análisis estadísticos pertinentes a los objetivos planteados en esta investigación, así como generalizar hasta cierto punto las conclusiones obtenidas, se consideró contar con, al menos, 10 encuestados por número de ítems (Muñiz, 2005). Se recogió información de 350 cuestionarios procedentes de 254 abuelos y abuelas, dado que algunos de ellos cuidan a más de un nieto o nieta y respondieron un cuestionario por cada uno de ellos ( $n=96$, $27.4 \%$ del total de cuestionarios recogidos). Para los abuelos y abuelas con más de un nieto o nieta a su cuidado, se incluyó el listado de preguntas por duplicado considerando que sus respuestas podrían ser distintas para cada uno de ellos. Los cuestionarios recogidos de los abuelos suponen el $39 \%$ de los 254 entregados, mientras que el porcentaje de las abuelas asciende al 61\%. El tamaño de la muestra garantiza contar con más de 
10 sujetos por ítem (24 ítems en el total del cuestionario), lo que permite realizar los análisis descriptivos y factoriales esperados.

\section{Análisis de datos}

Para dar respuesta al primer objetivo consistente en analizar la estructura y características técnicas de la escala de competencias emocionales y educativas de los abuelos y abuelas, se tuvieron en cuenta las especificaciones básicas de la Teoría Clásica del Test (TCT) (Gil, 2011; Muñiz, 2000). Se efectuó, en primer lugar, un análisis de las respuestas a los ítems para identificar: 1) datos perdidos y reemplazarlos a través de soluciones interactivas de sustitución con el algoritmo esperanzamaximización (Dempster, Laird \& Rubin, 1977; Pigott, 2001); 2) casos atípicos con tendencia hacia los valores extremos de la escala; 3) tendencia a la normalidad calculando media y desviación típica y valores de asimetría y curtosis, considerando que aquellos que superen el intervalo [3,10] se eliminarían del análisis (Kline, 2011); y 4) casos con puntuaciones atípicas multivariadas -outliers- a través del cálculo de la distancia de Mahalanobis $(p<.001)$. Se detectaron 4 casos que fueron eliminados del análisis, quedando la muestra compuesta por 346 personas. Posteriormente, se llevó a cabo un proceso de validación cruzada -cross-validation- con análisis factorial exploratorio (AFE) y análisis factorial confirmatorio (AFC) dividiendo la muestra inicial en dos secciones equivalentes de 173 sujetos cada una; ello permitiría garantizar que los factores obtenidos son independientes del grupo de sujetos sobre el que son calculados (Rao, Fung \& Rosales, 2008). Para realizar el AFE se comprobaron previamente los supuestos de aplicación del análisis mediante la medida de adecuación muestral de Kaiser-Meyer-Olkim (KMO) y el Test de Esfericidad de Bartlett. Después, se utilizó el método de Máxima Verosimilitud para la extracción de factores, seguido de rotación Promax para conseguir una mejor comprensión de la matriz resultante. Como método de retención de factores se utilizó K1, para retener sólo aquellos que tuvieran valores de eigenvalor superior a 1 (Kaiser, 1960; Fabrigar, Wegener, MacCallum \& Strahan, 1999); además, sólo se retuvieron ítems con cargas factoriales >
.40. Los datos se procesaron con el programa estadístico SPSS, 22.0. El AFC se llevó a cabo con el programa LISREL 8.80 a través del método de Máxima Verosimilitud, y teniendo en cuenta distintos índices de ajuste: Chi-Cuadrado; Normed Chi-Square, NC $(<3.0)$; Root Mean Square Error of Approximation, RMSEA (<.05); Standardised Root Mean Square Residual, SRMR (<.05); Comparative Fit Index, CFI (>.95); Normed Fit Index, NFI (>.95); y, por último, Tucker-Lewis Index, TLI $(>.95)$.

Para valorar la fiabilidad de la escala se analizó la consistencia interna para cada uno de los factores de forma independiente, mediante el índice de fiabilidad compuesta de Raykov (Raykov, 1997; Raykov \& Shrout, 2002). La validez concurrente se analizó calculando la correlación de Pearson entre los factores y las variables externas consideradas en este estudio.

Para dar respuesta al objetivo segundo de analizar las competencias emocionales y educativas de los abuelos y abuelas en función del sexo (hombre, mujer), la edad (40-64 años y más de 65 años) y la línea de parentesco (materna o paterna) se realizaron contrastes de grupos con la $t$ de Student para muestras independientes. En cada análisis se tuvo en cuenta si se cumplía la condición de homogeneidad de varianzas a través de la prueba de Levene y del estadístico F. En los casos en que las diferencias resultaron significativas se calculó el tamaño del efecto a través de la $\mathrm{d}$ de Cohen, considerando un tamaño bajo cuando $0<\mathrm{d}<.20$, medio-moderado: $.20<\mathrm{d}<.50$ y alto: $\mathrm{d}>.50$, (Cohen, 1988); si bien tenemos en cuenta que incluso un tamaño del efecto pequeño puede tener una significación práctica (Kirk, 1996).

\section{Resultados}

\section{Estructura y Calidad Técnica de la Escala de Competencias Emocionales de Abuelos y} Abuelas. Una vez analizadas las características de las respuestas a los 24 ítems iniciales, se seleccionaron 20 porque los 4 restantes no cumplieron el criterio de normalidad establecido con los valores de asimetría y curtosis. El AFE realizado con estos 20 ítems produjo una solución inicial de seis factores en la matriz de configuración a partir de los autovalores iniciales. 
Tabla 2. Estructura factorial de la Escala de Competencias Emocionales de Abuelos y Abuelas

\begin{tabular}{|c|c|c|c|c|}
\hline & $\begin{array}{l}\text { Factor } 1 \\
\text { Estrés }\end{array}$ & $\begin{array}{l}\text { Factor } 2 \\
\text { Seguridad }\end{array}$ & $\begin{array}{l}\text { Factor } 3 \\
\text { Dific.Conciliar }\end{array}$ & $\begin{array}{l}\text { Factor } 4 \\
\text { Autorregula }\end{array}$ \\
\hline $\mathrm{N}^{\mathrm{o}}$ Items Factor: & 3 & 2 & 3 & 2 \\
\hline$\%$ Varianza Total Explicada Factor & $13.83 \%$ & $11.37 \%$ & $10.73 \%$ & $9.30 \%$ \\
\hline $\begin{array}{l}\text { Items } \\
\text { Con mi nieto/a tengo la sensación de no } \\
\text { haber logrado todo lo que esperaba }\end{array}$ & \multicolumn{4}{|c|}{ Saturación } \\
\hline $\begin{array}{l}\text { Ser abuelo hace que yo me sienta nervioso } \\
\text { y agobiado }\end{array}$ & .72 & & & \\
\hline $\begin{array}{l}\text { Se me hace realmente difícil decidir cómo } \\
\text { hacer las cosas con mi nieto/a }\end{array}$ & .56 & & & \\
\hline $\begin{array}{l}\text { Creo que soy un buen abuelo y que } \\
\text { cumplo adecuadamente con mis funciones }\end{array}$ & & .67 & & \\
\hline $\begin{array}{l}\text { He conseguido ser tan buen abuelo como } \\
\text { yo quería }\end{array}$ & & .83 & & \\
\hline $\begin{array}{l}\text { Me siento agobiado por las circunstancias, } \\
\text { sin tiempo para poder ocuparme de mi } \\
\text { nieto/a }\end{array}$ & & & .67 & \\
\hline $\begin{array}{l}\text { Tener que cuidar de mi nieto/a me impide } \\
\text { hacer cosas o ir a sitios que me gustaría }\end{array}$ & & & .54 & \\
\hline $\begin{array}{l}\text { Siento que la responsabilidad de cuidar a } \\
\text { mi nieto/a no me deja disfrutar de él/ella } \\
\text { como me gustaría }\end{array}$ & & & .44 & \\
\hline $\begin{array}{l}\text { Cuando algo no sale como yo quiero con } \\
\text { mi nieto/a, procuro ver el lado positivo }\end{array}$ & & & & .61 \\
\hline $\begin{array}{l}\text { Sé relajarme y controlar mis emociones } \\
\text { ante mi nieto/a }\end{array}$ & & & & .63 \\
\hline
\end{tabular}

Todos los ítems obtuvieron pesos factoriales por encima del punto de corte establecido (.40); sin embargo, los factores 4 y 6 presentaron ítems con cargas factoriales relevantes en otros factores, por lo que sus ítems fueron eliminados. Una vez repetido el análisis se obtuvo una solución factorial con 10 ítems distribuido en cuatro factores (Tabla 2) que explicó el $45.25 \%$ de la varianza (medida de adecuación muestral de $\mathrm{KMO}=.720$; test de esfericidad de Bartlett $=671.3$ (190); $p<.001)$.

El primer factor, denominado "Estrés en la función de cuidado de los nietos y nietas", explica un $13.83 \%$ de la varianza y está compuesto por tres ítems: 1) "Con mi nieto/a tengo la sensación de no haber logrado todo lo que esperaba"; 2) "Ser abuelo/a hace que me sienta nervioso/a y agobiado/a"; y 3) "Se me hace realmente difícil decidir cómo hacer las cosas con mi nieto/a". El segundo, denominado "Seguridad personal en la función de cuidado de sus nietos y nietas", explica el $11.37 \%$ de la varianza y está compuesto por dos ítems: 1) "Creo que soy un/a buen/a abuelo/a y que cumplo adecuadamente con mis funciones"; y 2) "He conseguido ser tan buen/a abuelo/a como yo quería". El tercero, denominado "Dificultad para conciliar las circunstancias personales y el rol de abuelo y abuela", explica el $10.73 \%$ de la varianza y está compuesto por tres ítems: 1) "Me siento agobiado/a por las circunstancias, sin tiempo para poder ocuparme de mi nieto/a"; 2) "Tener que cuidar de mi nieto/a me impide hacer cosas o ir a sitios que me gustaría"; y 3 ) "Siento que la responsabilidad de cuidar a mi nieto/a no me deja disfrutar de él -o ella- como me gustaría". Por último, el cuarto factor, denominado "Autorregulación emocional en el ejercicio del rol de abuelo y abuela", explica el $9.30 \%$ de la varianza y está compuesto por dos ítems: 1) "Cuando algo no sale como yo quiero con mi nieto/a, procuro ver el lado positivo"; y 2) "Sé relajarme y controlar mis emociones ante mi nieto/a". 
.52

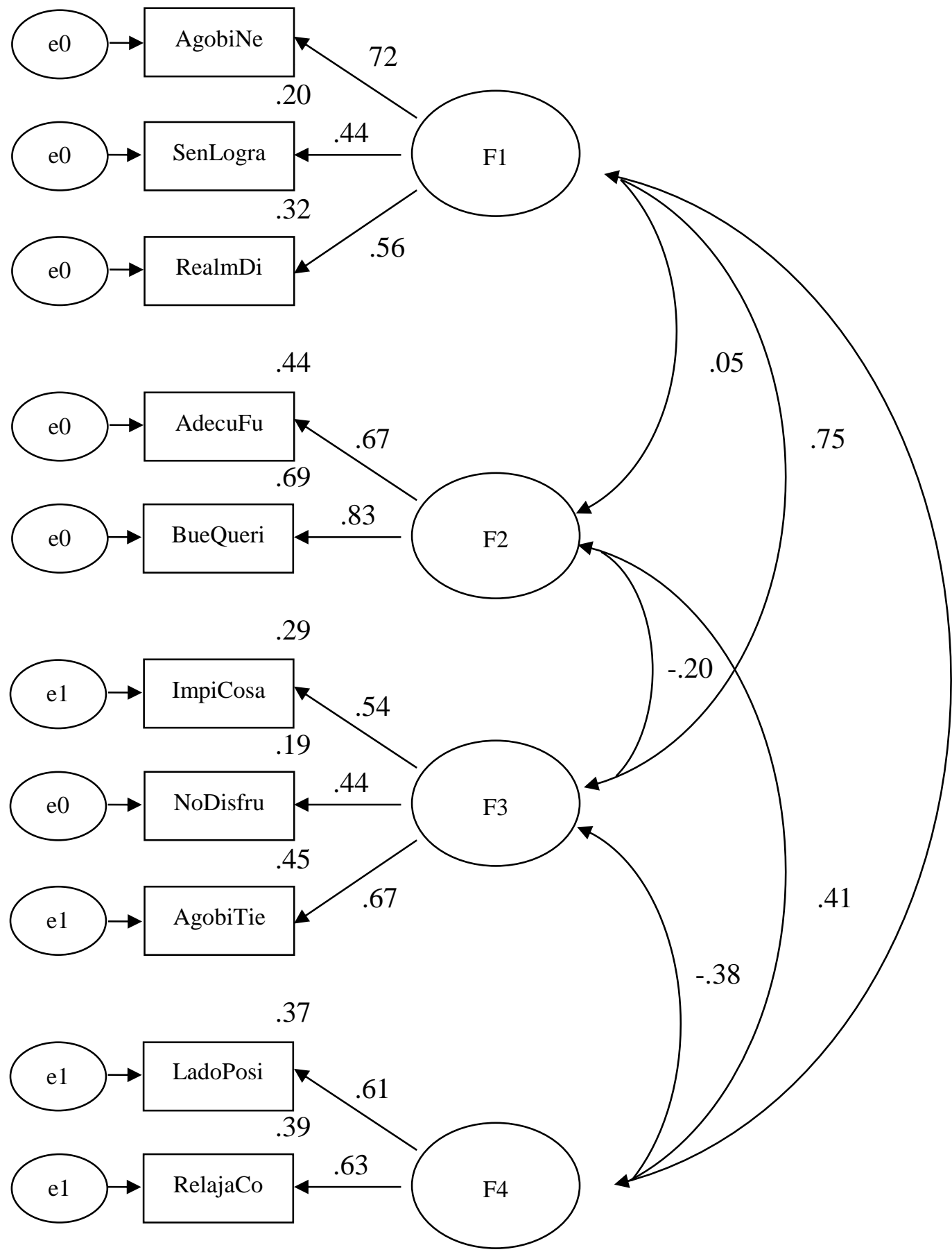

Figura 1: Análisis Factorial Confirmatorio

Tras efectuar el AFE, se llevó a cabo con la segunda submuestra un proceso de validación cruzada con el AFC (Figura 1). Los índices de ajuste obtenidos confirmaron un modelo compuesto por 10 ítems distribuidos en cuatro factores: Chi-cuadrado $\quad(29)=31.02, \quad p=.36$; $\mathrm{NC}=1.07 ; \quad \mathrm{RMSEA}=.020, \quad$ pclose $=.902$; $\mathrm{SRMR}=.041 ; \mathrm{NFI}=.953 ; \mathrm{NNFI}=.987$ y CFI=.991.

La fiabilidad obtenida en cada factor, calculada a través del índice compuesto de Raykov, resultó aceptable: F1: .62; F2: .72; F3: .61; F4: .68.

Complementariamente, se ha analizado la relación entre los factores a través de la correlación de Pearson, encontrando que el Factor 1-Estrés correlaciona positivamente con el Factor 3-DificultadConciliación, ( $\mathrm{r}=.369, \quad p=.000) \quad \mathrm{y}$ negativamente con el Factor 4-Autorregulación $(\mathrm{r}=-.226, p=.000)$. Por su parte, el Factor 2Seguridad, correlaciona negativamente con el Factor 3-DificultadConciliación ( $\mathrm{r}=-.189, p=.001$ ) y positivamente con el Factor 4-Autorregulación 
Tabla 3. Correlaciones significativas entre los factores y las variables criterio externas

\begin{tabular}{lcccccc}
\hline & $\begin{array}{c}\text { F1- } \\
\text { Estrés }\end{array}$ & $\begin{array}{c}\text { F2- } \\
\text { Seguridad }\end{array}$ & $\begin{array}{c}\text { F3- } \\
\text { DifConciliar }\end{array}$ & $\begin{array}{c}\text { F4- } \\
\text { Autorregu }\end{array}$ & Eficacia & Satisfacción \\
\hline F1-Estrés & 1 & & & & & \\
F2-Seguridad & ---- & 1 & & & & \\
F3- & $.369^{* *}$ & $-.189^{* *}$ & 1 & & & \\
Dific.Conciliar & $-.226^{* *}$ & $.299^{* *}$ & $-.221^{* *}$ & 1 & & \\
F4-Autorregula &.--- & $.398^{* *}$ & --- & $.311^{* *}$ & 1 & \\
Eficacia & $-----9^{* *}$ & --- & & 1 \\
Satisfacción & $-.350^{* *}$ & --- & $-.299^{*}$ & & & \\
\hline$* p \leq .05$ & & & & & & \\
$* * p \leq .01$ & & & & &
\end{tabular}

$(\mathrm{r}=.299, \quad p=.000) . \quad$ Asimismo, este último correlaciona negativamente con el Factor 3DificultadConciliación $(\mathrm{r}=-.221, \quad p=.000)$. El análisis de la validez concurrente calculada mediante la correlación entre cada factor y las variables criterio externas al cuestionario, indica que el ítem "Me manejo muy bien con las tareas que tengo que hacer con mi nieto/a" (eficacia), obtiene una correlación con el Factor 2Seguridad $=.398 \quad(p=.000)$ y con el Factor 4Autorregulación $=.311 \quad(p=.000)$. Asimismo, el ítem "Hacer de abuelo/a es tan satisfactorio como yo esperaba" (satisfacción) alcanza una correlación con el Factor 1-Estrés=-.350 ( $p=.000)$ $y$ con el Factor 3-DificultadConciliación=-.299 $(p=.000)$.

\section{Competencias emocionales de los abuelos y} abuelas en función del sexo, la edad y la línea de parentesco. Para comprobar la posible influencia del sexo, la edad y la línea de parentesco sobre las competencias emocionales y educativas de los abuelos y abuelas, se han efectuado contrastes de grupos en cada factor y se ha calculado el tamaño del efecto cuando las

diferencias resultaron significativas. Con respecto al primer factor, relativo al estrés que puede suscitar al abuelo y abuela el cuidado y educación de su nieto o nieta, los datos indican que la mayoría no siente estrés (media=1.39; DT=.510); el análisis del segundo factor indica que la mayoría de los abuelos y abuelas que han participado en este estudio se sienten seguros al desempeñar la función de cuidado y educación con sus nietos y nietas (media=3.40; DT=.644). En torno al tercer factor, sobre las dificultades de conciliación entre sus circunstancias personales y el desarrollo del rol de abuelo y abuela, los resultados indican que la mayoría de participantes en el estudio tienen pocas dificultades (media=1.37; DT=.463) y en cuanto al factor cuarto, relativo a la autorregulación emocional de los abuelos y abuelas en el desempeño de este rol, la mayoría de la muestra indica niveles adecuados (media=3.15; DT=.691).

Con respecto al sexo, se han encontrado diferencias significativas sólo en el Factor 2Seguridad (Tabla 4), con un tamaño del efecto medio ( $\mathrm{t}(334)=1.989 ; p=.048, \mathrm{~d}=.22)$, sugiriendo que las abuelas tienen más confianza en sí mismas que los abuelos en el desempeño de su rol educativo con los nietos y nietas. En los restantes factores estas diferencias no se han encontrado. Por su parte, la edad no parece incidir diferencialmente en ninguno de los factores considerados. La línea de parentesco, sin embargo, sí establece diferencias en el Factor 2Seguridad (Tabla 4), con un tamaño del efecto medio ( $\mathrm{t}(334)=3.180 ; p=.002, \mathrm{~d}=.35)$, indicando que los abuelos y abuelas paternos tienen más seguridad que los maternos. En los otros factores, no se aprecian diferencias relevantes.

\section{Discusión y Conclusiones}

La familia es un contexto clave en el desarrollo integral y en la estabilidad emocional y afectiva de sus miembros más pequeños, siendo los padres y madres los principales agentes de su socialización. No obstante, en la actualidad muchos tienden a delegar en los abuelos y abuelas parte de sus funciones de crianza y educación de sus hijos e hijas por diversas razones (cambios en la estructura familiar, limitación de tiempo, dificultades para conciliar la vida laboral, familiar y personal, etc.). A este respecto, autores como Buz y Bueno (2006), Megías y Ballesteros (2011), 
Tabla 4. Diferencias estadísticamente significativas en los factores de competencias emocionales de los abuelos y abuelas en función del sexo y de la línea de parentesco

\begin{tabular}{|c|c|c|c|c|c|c|}
\hline & & $\mathrm{N}$ & $\begin{array}{c}\mathrm{M} \\
\text { (DT) }\end{array}$ & $\mathrm{t}$ & $p$ & d \\
\hline \multicolumn{7}{|l|}{ Diferencias en función del sexo } \\
\hline \multirow{2}{*}{ F2-Seguridad } & $\mathrm{M}$ & 198 & $\begin{array}{l}3.45 \\
(.63)\end{array}$ & 1.989 & .048 & .22 \\
\hline & $\mathrm{H}$ & 136 & $\begin{array}{l}3.31 \\
(.65)\end{array}$ & & & \\
\hline \multicolumn{7}{|l|}{$\begin{array}{l}\text { Diferencias en función de la línea de } \\
\text { parentesco }\end{array}$} \\
\hline \multirow{2}{*}{ F2-Seguridad } & $\mathrm{P}$ & 131 & $\begin{array}{l}3.53 \\
(.59)\end{array}$ & \multirow[t]{2}{*}{3.180} & \multirow[t]{2}{*}{.002} & \multirow[t]{2}{*}{.35} \\
\hline & M & 203 & $\begin{array}{l}3.31 \\
(.66)\end{array}$ & & & \\
\hline
\end{tabular}

Nota. $\mathrm{M}=$ Mujer; $\mathrm{H}=$ Hombre

Nota. $\mathrm{P}=$ Paterna; $\mathrm{M}=$ Materna

entre otros, entienden que hoy, en la familia, los abuelos y abuelas están mucho más presentes que en épocas pasadas, convirtiéndose la actividad que desempeñan para con sus nietos y nietas en irremplazable. Con esa actividad adquieren nuevas e importantes responsabilidades que superan las que tradicionalmente se esperaba de ellos, basadas en proporcionar afecto, apoyo emocional y seguridad a sus nietos y nietas (Bernad, 2012). Por ello, se entiende que necesitan contar con competencias emocionales y educativas que les faciliten el desempeño de este rol. La valoración de estas competencias, no obstante, se ve dificultada por la escasez de técnicas validadas en nuestro país de recogida de información al respecto; de ahí que en la presente investigación se haya diseñado una escala que permita profundizar en estos aspectos. Para ello, se ha contado con una muestra suficientemente amplia y con características sociodemográficas diversas.

La estructura factorial resultante de la escala diseñada se compone de cuatro factores que aglutinan 10 ítems sobre F1-Estrés en la función de cuidado y educación de los nietos y nietas, F2Seguridad personal en el ejercicio de esas funciones, F3-Dificultad para conciliar esta tarea con la situación personal, y F4-Autorregulación emocional en el ejercicio de la misma. Los resultados descriptivos obtenidos sobre cada factor indican que la muestra estudiada se percibe en su mayoría con competencias adecuadas para desarrollar su rol educativo con sus nietos y nietas, con seguridad, sin nerviosismo ni agobio. En este sentido, Triadó, Villar, Celdrán y Solé (2014) y Triadó et al. (2009) hacen alusión a sentimientos de disfrute y satisfacción; así pues, la ausencia de estrés y la seguridad que presentan, les facilita desenvolverse adecuadamente en su cometido educativo con los nietos y nietas, percibiéndose a sí mismos como "buenos" abuelos y abuelas; en la muestra de este estudio esa competencia de seguridad personal la tienen significativamente más desarrollada las abuelas que los abuelos. La diferencia encontrada quizá pueda deberse a la mayor asiduidad con que ellas se hacen cargo de este tipo de tareas familiares, tal y como Mestre, Guillen y Caro (2012), Micolta y Escobar (2010), Pérez (2007) y Villalba (2002) recogen en sus trabajos. De igual modo, los abuelos y abuelas paternos presentan en este estudio un mayor nivel de seguridad que los maternos, posiblemente porque la implicación de estos últimos sea más frecuente y exigente, tal como expresan Mestre (2012), Osuna (2006) y Rico, Serra, Viguer y Meléndez (2000), y les haga ser más vulnerables.

Dada esa valoración positiva que estos abuelos y abuelas tienen de sí mismos, muestran un bajo nivel de estrés y una elevada seguridad en el desarrollo de su función educativa, lo que parece que se traduce en desenvoltura y en una organización adecuada para conciliar esta función educativa con otras actividades personales, permitiéndoles, como ya se ha adelantado, disfrutar de su rol de abuelos y abuelas sin sentimientos negativos. En consonancia, parece que tienen habilidades de relajación y de autorregulación emocional apropiadas, pues la mayoría sabe controlarse ante sus nietos y nietas; lo que también hace que den un enfoque positivo cuando algo no sale como quieren con ellos. En 
sus contribuciones, Hoyuelos (2004), Osuna (2006), Triadó et al. (2014) y Villalba (2002), hacen hincapié en ese optimismo que vincula el disfrute y satisfacción que sienten al estar con sus nietos y nietas.

Al considerar la edad de los abuelos y abuelas no se han encontrado en este estudio diferencias significativas en ninguno de los factores analizados, lo que contradice lo indicado por Bernad (2012), Osuna (2006) y Rico, Serra y Viguer (2001), quienes señalan la edad de los abuelos y abuelas como un posible condicionante del rol que desempeñan.

Por su parte, las relaciones entre los factores identificados en este estudio indican que: 1) cuanta más dificultad encuentren los abuelos y abuelas para conciliar el cuidado y educación de sus nietos y nietas con su vida personal, más estrés sienten; 2) su grado de estrés es mayor en la medida en que cuenten con menos habilidades de autorregulación; 3) cuanta más seguridad tienen en sí mismos, menos dificultad perciben en conciliar su cometido y su actividad personal; 4) cuanta más seguridad tienen, más capaces son de autorregular sus emociones; 5) a más capacidad de autorregulación, menos dificultad encuentran en torno a la conciliación; y viceversa. Por tanto, como implicaciones para la práctica, parece conveniente que los abuelos y abuelas que se hagan cargo del cuidado de sus nietos y nietas tengan circunstancias adecuadas para poder conciliar su tiempo de dedicación a sus nietos y nietas con sus otras responsabilidades y actividades personales, de modo que puedan desarrollarlas sin estrés. En todo caso, si esta circunstancia no fuera posible, conviene que tengan adquirida seguridad en sí mismos para poder regular mejor la posible tensión que puedan sentir por las dificultades de conciliación.

Los resultados de este trabajo han permitido identificar cuatro aspectos relevantes que inciden en la dinámica cotidiana de los abuelos y abuelas que asumen el cuidado y educación de sus nietos y nietas: el estrés al desarrollar esta función, la seguridad personal, la dificultad para conciliarla con su situación personal y la autorregulación emocional. Así pues, y siendo escasa la información empírica que existe en España sobre la temática estudiada, este trabajo aporta algunas conclusiones relevantes sobre cómo perciben los abuelos y abuelas su rol educativo y de cuidado con sus nietos y nietas. En general, esta percepción es positiva; no obstante es relevante considerar también los casos en que los abuelos y abuelas señalan dificultades con el fin de trabajar sobre ello en posibles líneas de orientación educativa familiar que respondan a su necesidad de apoyo y de formación. Este estudio aporta también una escala validada, de fácil aplicación e interpretación que puede facilitar seguir indagando sobre las competencias emocionales y educativas de este colectivo.

Entre las limitaciones de este estudio a considerar en futuras investigaciones cabe citar no contar con una muestra aleatoria representativa de la población y con un tamaño muestral más grande, que permitan una mayor generalización de los resultados obtenidos. A efectos de profundizar más en la validación de la escala, sería conveniente contar con datos aplicados en otras escalas similares. Asimismo, sería interesante contar también con información cualitativa procedente de entrevistas o de grupos de discusión para contrastar con más detalle las conclusiones obtenidas.

\section{Referencias}

Badenes, N., \& López, M. T. (2011). Doble dependencia: Abuelos que cuidan nietos en España. Zerbitzuan, 49, 107-125. doi:10.5569/1134-7147.49.09

Bernad, J. A. (2012). De profesión, abuelo. Ser abuelo hoy (...con vocación y fundamento). Barcelona: Editorial Graó.

Bisquerra, R., \& Pérez, N. (2007). Las competencias emocionales. Educación XXI, 21(10), 61-82. doi:10.5944/educxx1.1.10.297

Buz, J., \& Bueno, B. (2006). Las relaciones intergeneracionales. Lecciones de Gerontología, 66. Madrid: Informes Portal Mayores.

Cabero, J., \& Infante, A. (2014). Empleo del método delphi y su empleo en la investigación y comunicación científica. EDUTEC: Revista Electrónica de Tecnología Educativa, 48, 116. doi:10.21556/edutec.2014.48.187

Cherlin, A., \& Furstenberg, F. (1985). Styles and strategies of grandparenting. En V. L. Bengston \& J. F. Robertson (Eds.), 
Grandparenthood (pp.97-116). Beverly Hills: Sage Publications.

Cherlin, A., \& Furstenberg, F. (1986). The new American grandparent: A place in the family, a life apart. New York: Basic Books.

Cohen, J. (1988). Statistical power analysis for the behavioural science. (2nd ed.). Hillsdale, NJ: Lawrence Erlbaum Associates.

De Lara-Guijarro, E., \& Ballesteros-Velázquez, B. (2007). Métodos de investigación en Educación Social. Madrid: Universidad Nacional de Educación a Distancia.

Dempster, A. P., Laird, N. M., \& Rubin, D.B. (1977). Maximum likelihood from incomplete data via the EM algorithm. Journal of the Royal Statistical Society, Series B. 39(1), 1-38.

Fabrigar, L. R., Wegener, D. T., MacCallum, R. C., \& Strahan, E. J. (1999). Evaluating the use of exploratory factor analysis in psychological research. Psychological Methods, 3, 272-299. doi:10.1037/1082-989X.4.3.272

Farkas, C. (2008). Escala de Evaluación Parental (EEP): Desarrollo, propiedades psicométricas y aplicaciones. Universitas Psychologica, 7(2), 457-467.

Frías, M. T., Díaz, R., \& Reyes, I. (2011). Desarrollo y validación de una escala para medir apoyo social en adultos con perspectiva de apego. Revista Iberoamericana de Diagnóstico y Evaluación Psicológica - e Avaliação Psicológica, 2(32), 9-34.

García, V., Pérez, M. H., \& Martínez, R. A. (2014). Aproximación a la participación de los abuelos y abuelas en la educación de sus nietos y nietas. INFAD Revista de Psicología. International Journal of Developmental and Educational Psychology, 1(1), 571-580. doi:10.17060/ijodaep.2014.n1.v1.405

Gil, J. A. (2011). Técnicas e instrumentos para la recogida de información. Madrid: UNED.

Gimeno, A. (1999). La familia: El desafío a la diversidad. Barcelona: Ariel.

Gomila, M. (2005). Las relaciones intergeneracionales en el marco de la familia contemporánea: Cambios y continuidades en transición hacia una nueva concepción de la familia. Historia contemporánea, 31, 505542.
González, J. (2007). Características de los abuelos del Siglo XXI. INFAD Revista de Psicología. International Journal of Developmental and Educational Psychology, 2, 29-42.

González, J. De la Fuente, R., \& González, J. (2015) Abuelo y nieto, vínculo simbólico. En S. Rivas (Coord.), Generaciones conectadas. Beneficios educativos derivados de la relación entre nietos y abuelos (pp. 105-122). Madrid: Ediciones Pirámide.

González, J. González, J., De la Fuente, R., Marquínez, S., \& González, N. (2010). Funciones que desempeñan los abuelos. INFAD Revista de Psicología. International Journal of Developmental and Educational Psychology, 2, 625-633.

Goodman, L. A. (1961). Snowball Sampling. Annals of Mathematical Statistics, 32, 148170.

Hoyuelos, A. (2004). Abuelos, abuelas, nietos y nietas. El punto de vista infantil. Indivisa, Bol. Estud. Invest., 5, 35-42.

Iglesias de Ussel, J. (1998). La familia española en el contexto europeo. En M. J. Rodrigo y J. Palacios (Coords.), Familia y desarrollo humano (pp. 91-114). Madrid: Alianza Editorial.

Isaza, L. (2013). Una aproximación a los contextos familiar y escolar como posibilitadores del desarrollo social. Realitas: Revista de Ciencias Sociales, Humanas y Artes, 1(2), 39-45.

Johnston, C., \& Mash, E. J. (1989). A measure of parenting satisfaction and efficacy. Journal of Clinical and Child Psychology, 18(2), 167175. doi:10.1207/s15374424jccp1802_8

Kaiser, H. F. (1960). The application of electronic computers to factor analysis. Educational and Psychological Measurement, 20, 141-151. doi:10.1177/001316446002000116

Kirk, R. E. (1996). Practical significance: A concept whose time has come. Educational \& Psychological Measurement, 56, 746-759. doi:10.1177/0013164496056005002

Kline, R. B. (2011). Principles and practice of structural equation modeling. (5th ed.). New York: The Guilford Press.

León, O. G., \& Montero, I. (2003). Métodos de investigación en psicología y educación. $\left(3^{\mathrm{a}}\right.$ ed.). Madrid: McGraw-Hill. 
Maglio, A. L., Molina, M. F., Raimundi, M. J., González, M. A., \& Schmidt, V. (2014). El apoyo familiar en la interfaz familia-trabajo. Construcción de una escala para su evaluación. Revista Iberoamericana de Diagnóstico y Evaluación Psicológica - e Avaliação Psicológica, 1(37), 187-202.

Martínez, R. A. (1996). Familia y educación. Fundamentos teóricos y metodológicos. Universidad de Oviedo: Servicio de Publicaciones.

Martínez, R. A. (2009). Programa-Guía para el desarrollo de competencias emocionales, educativas y parentales. Madrid: Ministerio de Sanidad y Política Social.

Megías, I., \& Ballesteros, J. C. (2011). Abuelos y abuelas... para todo: Percepciones en torno a la educación y el cuidado de los nietos. Madrid: FAD.

Meil, G., \& Rogero-García, J (2014) Abuelas, abuelos y padres varones en el cuidado de la infancia. Cuadernos de Relaciones Laborales, 32(1), 49-67. doi:10.5209/rev_CRLA.2014.v32.n1.44713

Menéndez, S. (2001a). Diversidad familiar y desarrollo psicológico infantil. Portularia, 1, 215-222.

Menéndez, S. (2001b). La diversidad familiar en España. Un análisis de su evolución reciente y su aceptación. Apuntes de Psicología, 19(3), 367-386.

Mestre, J. M. (2011). Repercusión de la conciliación de la vida social y familiar en las abuelas cuidadoras en el siglo XXI. Ponencia, III Congreso Anual de la REPS. Los actores de las políticas sociales en un contexto de transformación. Universidad Pública de Navarra, Pamplona.

Mestre, J. M., Guillen, J., \& Caro, F. (2012). Abuelas cuidadoras en el Siglo XXI: Recurso de conciliación de la vida social y familiar. Portularia, 12(extra), 231-238. doi:10.5218/prts.2012.0025

Micolta, A., \& Escobar, M. C. (2010). Si las abuelas se disponen a cuidar, madres y padres pueden emigrar. Revista Venezolana de Estudios de la Mujer, 15(35), 91-115.

Muñiz, J. (2000). Teoría clásica de los tests. Madrid: Pirámide.
Muñiz, J. (2005). Classical test models. En B.S. Everitt and D. C. Howell (Ed.). Encyclopedia of Statistics in Behavioral Science (pp. 278282). Chichester, UK: John Wiley \& Sons Ltd.

Muñoz, A. (2005). La familia como contexto de desarrollo infantil. Dimensiones de análisis relevantes para la intervención educativa y social. Portularia, 5(2), 147-163.

Neugarten, B. L., \& Weinstein, K. K. (1964). The changing American grandparent. Journal of Marriage and the Family, 45, 267-276. doi:10.2307/349727

Osuna, M. J. (2006). Relaciones familiares en la vejez: Vínculos de los abuelos y de las abuelas con sus nietos y nietas en la infancia. Revista Multidisciplinar de Gerontología, 16(1), 16-25.

Palacios, J., \& Rodrigo, M. J. (1998). La familia como contexto de desarrollo humano. En M. J. Rodrigo y J. Palacios (Coords.), Familia y desarrollo humano (pp. 25-44). Madrid: Alianza Editorial.

Perea, R. (2006). La familia como contexto para un desarrollo saludable. REP: Revista Revista Española de Pedagogía, 235, 417428.

Pérez, L. (2007). Las abuelas como recurso de conciliación entre la vida familiar y laboral. Presente y futuro. Madrid: Instituto de la Mujer, Ministerio de Trabajo y Asuntos Sociales.

Pigott, T. D. (2001). A review of methods for missing data. Educational Research and Evaluation, 7(4), 353-383.

Pinazo, S. (1999). El significado social del rol del abuelo. Revista Multidisciplinar de Gerontología, 9, 169-176.

Rao, R. B., Fung, G., \& Rosales, R. (2008). On the dangers of cross-validation. An experimental evaluation. In Proceedings of the SIAM International Conference on Data Mining (SDM) (pp. 588-596). Philadelphia, PA: SIAM. doi:10.1137/1.9781611972788.54

Raykov, T. (1997). Estimation of composite reliability for congeneric measures. Applied Psychological Measurement, 21(2), 173-184. doi:10.1177/01466216970212006

Raykov, T., \& Shrout, P. E. (2002). Reliability of scales with general structure: Point and 
interval estimation using a structural equation modeling approach. Structural Equation Modeling, 9(2), 195-212. doi:10.1207/S15328007SEM0902_3

Rico, C., Serra, E., \& Viguer, P. (2001). Abuelos y nietos: abuelo preferido, abuelo útil. Madrid: Pirámide.

Rico, C., Serra, E., Viguer, P., \& Meléndez, J. C. (2000). Las relaciones abuelos-nietos al final del milenio: La visión de los niños. Geriátrika, 16(9), 329-336.

Roa, J. M., \& Vacas, C. (2000). Perfiles de abuelidad. Pedagogía Social: Revista Interuniversitaria, 6-7, 205-219.

Rodríguez, I., \& Menéndez, S. (2003). El reto de las nuevas realidades familiares. Portularia, 3 , 9-32.

Tobío, C. (2002) Conciliación o contradicción: cómo hacen las madres trabajadoras. REIS: Revista Española de Investigaciones Sociológicas, $\quad 97, \quad$ 155-186. doi: $10.2307 / 40184368$

Tobío, C. (2012). Reciprocity and solidarity in intergenerational relationships: Spain, France and Norway in comparative perspective. Papers, 97(4), 849-873. doi:10.5565/rev/papers/v97n4.246

Tobío, C. (2013). Estado y familia en el cuidado de las personas: sustitución o complemento. Cuaderno de Relaciones Laborales, 31(1), 1738. doi:10.5209/rev_CRLA.2013.v31.n1.41623

Triadó, C. (2015) El rol de los abuelos en la estructura familiar actual. En S. Rivas (Coord.), Generaciones conectadas. Beneficios educativos derivados de la relación entre nietos y abuelos (pp. 59-79). Madrid: Ediciones Pirámide.

Triadó, C., Celdrán, M., Conde, L, Montoro, J., Pinazo, S., \& Villar, F. (2008a). Envejecimiento productivo. La provisión de cuidados de los abuelos a los nietos. Implicaciones para su salud y bienestar. Informe de investigación IMSERSO. Madrid: IMSERSO.

Triadó, C., Martínez, G., \& Villar, F. (2000). El rol y la importancia de los abuelos para sus nietos adolescentes. Anuario de Psicología, 31(2), 107-118.

Triadó, C., Villar, F., Celdrán, M., \& Solé, C. (2014). Grandparents who provide auxiliary care for their grandchildren: Satisfaction, difficulties, and impact on their health and wellbeing. Journal of Intergenerational Relationships, 12(2), 113127. doi:10.1080/15350770.2014.901102

Triadó, C., Villar, F., Pinazo, S., Solé, C., Montoro, J., \& Celdrán, M. (2008b). La relación entre abuelos/as y sus nietos/as adolescentes: comparación de perspectivas generacionales. Infancia y aprendizaje, 31(3), 385-398. doi:10.1174/021037008785702938

Triadó, C., Villar, F., Solé, C., Osuna, M. J., \& Pinazo, S. (2005). The meaning of grandparenthood: Do adolescent grandchildren perceive the relationship and role in the same way their grandparents do? Journal of International Relationships, 3(2), 101-121. doi:10.1300/J194v03n02_07

Triadó, C., Villar, F., Solé, C., Celdrán, M., Pinazo, S., Conde, L., \& Montoro, J. (2008c). Las abuelas/os cuidadores de sus nietos/os: Tareas de cuidado, beneficios y dificultados del rol. INFAD Revista de Psicología. International Journal of Developmental and Educational Psychology, 4(1), 455-464.

Triadó, C., Villar, F., Solé, C., Celdrán, M., Pinazo, S., \& Conde, L. (2009). Los abuelos/os cuidadores de sus nietos: Percepción de ayudas recibidas, conductas problemáticas de los nietos y satisfacción con el rol. INFAD Revista de Psicología. International Journal of Developmental and Education Psychology, 3(1), 497-506.

Valdivia, C. (2008). La familia: Concepto, cambios y nuevos modelos. La Revue $d u$ REDIF, 1, 15-22.

Van Rast, N., Verschueren, K., \& Marcoen, A. (1995). The meaning of grandparents as viewed by adolescent grandchildren: An empirical study in Belgium. International Journal of Aging and Human Development, 41(3), 11-324. doi:10.2190/PPLV-DWG7HV9R-6LVB

Villalba, C. (2002). Abuelas cuidadoras: Una aportación para el trabajo social. Valencia: Tirant lo Blanch.

Wood, V., \& Robertson, J. F. (1976). The significance of grandparenthood. In J. F. Gubrium (Ed.). Time, roles, and self in old age (pp. 278-304). New York: Human Sciences Press. 